\title{
Red capes and physician resilience
}

$\mathrm{P}$ erhaps physicians should trade in their white coats for red capes. Many seem to think they possess otherworldly strength, at least with respect to their resilience to stress and burnout.

"They often portrayed themselves as superhuman. ... They spoke as if they were immune to stress," Alicia Polachek, a researcher with Ward of the 21st Century, an innovation initiative at the University of Calgary in Alberta, said Oct. 26 during a presentation on physician resilience at the International Conference on Physician Health in Montréal, Quebec.

"Research suggests these attitudes are likely due, in part, to the culture of medicine," said Polachek, noting that doctors are taught to always be in control and to strive for perfection.

That culture isn't kind to doctors who show signs of being affected by stress. Colleagues generally perceive them not as unhealthy, but as weak, one doctor in the audience suggested. Instead of receiving support, they are stigmatized.

Researchers say they're scrambling to comprehend why some doctors appear resilient and immune to stress, and others not. Even the notion of resilience is up for grabs.

"Resilience, the concept, is in its infancy in terms of the medical profession and how it fits into wellness," says Dr. Jane Lemaire, clinical professor of medicine and vice chair of physician wellness and vitality in the Faculty of Medicine at the University of Calgary.

A group of Calgary researchers searched scientific literature for mentions of "physician" and "resilience" over the past two years and found a mere four or five references, and "only two that truly had any substance," Lemaire says.

The concept is so new that it would be premature to even define it, says Dr. Mamta Gautam, an Ottawa psychiatrist and expert in physician health and wellbeing. "We can't just make up a defini-

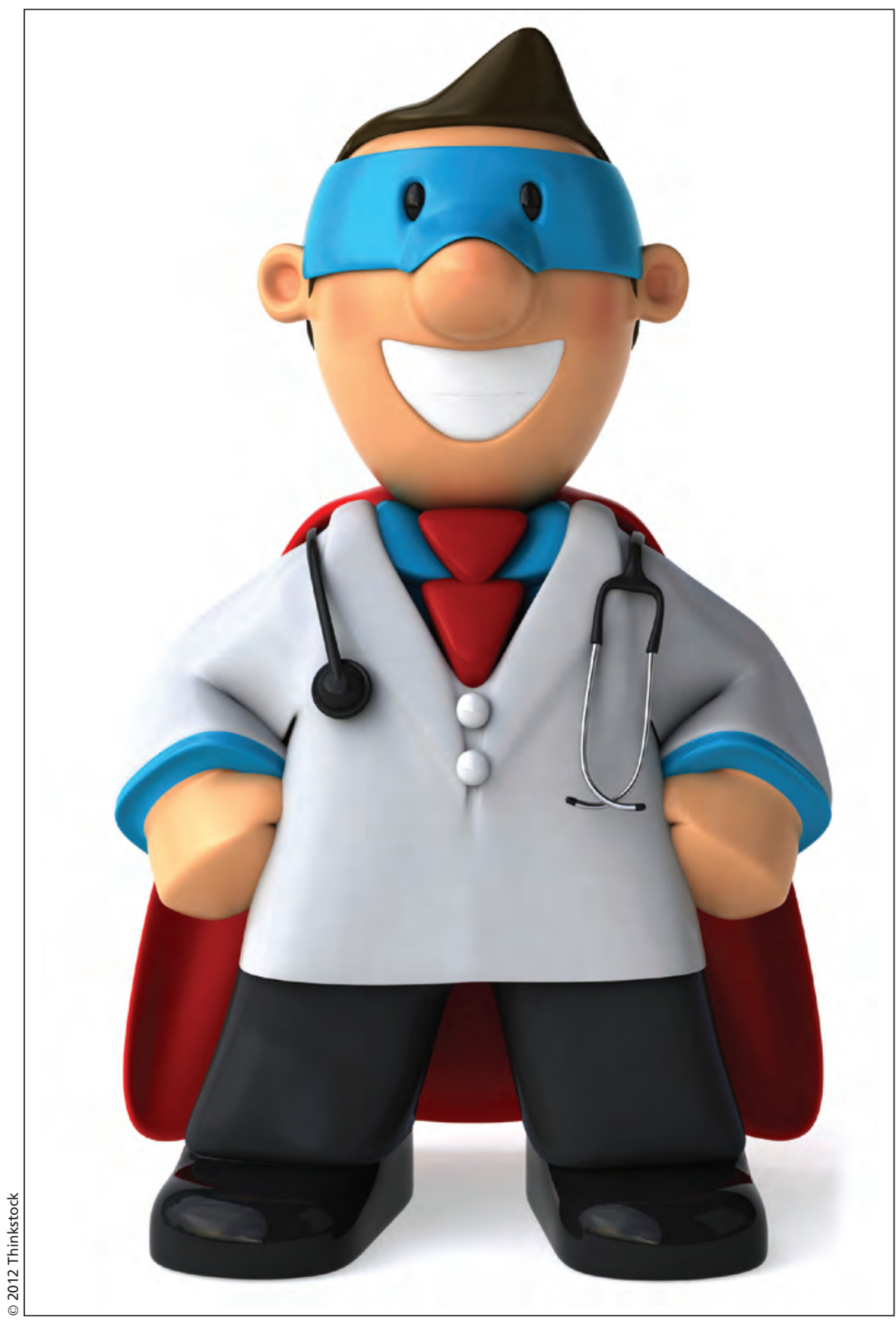

Many physicians seem to think they possess otherworldly strength, at least with respect to their resilience to stress and burnout.

tion," she says. "We really have to go back to laying the groundwork and understanding the concept."

Beyond that lies the pedagogical challenge of teaching resilience. While half of all physicians experience burnout, that means half don't, and researchers hope to ascertain why the luckier few cope well with stress. Moreover, "how do we go about teaching it in a meaningful way?" says Lemaire.

Such information would be invalu- 
able to burnt out physicians, Gautam says. "I've seen physicians in my practice for more than 20 years, and I'm able to see trends. Doctors are asking for more proactive ways to stay well in medicine."

Gautam and colleagues surveyed physicians for their views on the concept of resilience. The responses indicated that physicians believe many factors contribute to resilience, including stage of life, professional experience, personality traits, coping skills, level of social support and quality of personal relationships.
Opinions were split about whether resilience is innate or learned. Some believed it could be augmented by upbringing, education and medical training. Others believed it was learned through mentorship, shared experiences and coping strategies.

Barriers to physicians becoming more resilient to stress include self-perceptions of resilience (though respondents often didn't think the same of all their colleagues), lack of awareness and insight, and "ego resistance to improving their personal health circumstances," Gautam told the gathering.
While the preliminary research could lead to the development of tools to measure potential resilience levels, those aren't expected soon. "To develop a measure and then validate it in a large population takes five or six years of research," says Lemaire. "It's a lot of work. And it's hard work."

But it would be of enormous benefit, Gautam notes. "You can learn how to modify how you act so it's healthier for you and the people around you." Roger Collier, CMAJ

CMAJ 2012. DOI:10.1503/cmaj.109-4331 